\title{
Why has catchment evaporation increased in the past 40 years? A data-based study in Austria
}

Doris Duethmann ${ }^{1}$, Günter Blöschl ${ }^{1}$

$5{ }^{1}$ Institute for Hydraulic and Water Resources Engineering, Vienna University of Technology, Karlsplatz 13/223, A-1040 Vienna, Austria.

Correspondence to: Doris Duethmann (duethmann@hydro.tuwien.ac.at) 


\section{Wind data}

Wind data were regarded as not representative with respect to trends. The reasons for this are: i) annual anomalies of wind speed data from 85 stations in Austria appear unrelated to each other (Supplementary Figure S 1a) and temporal trends over 1977-2014 do not show any spatial pattern (Supplementary Figure S 2a); ii) averaged anomalies of annual wind speeds from station data and ERA Interim data (Dee et al., 2011) show for most part of the series opposing patterns (Supplementary Figure S 2); and iii) wind data are known to be prone to inhomogeneities (Böhm, 2008). We therefore used uniform monthly wind speeds averaged over all years and over all stations in Austria.

(a)

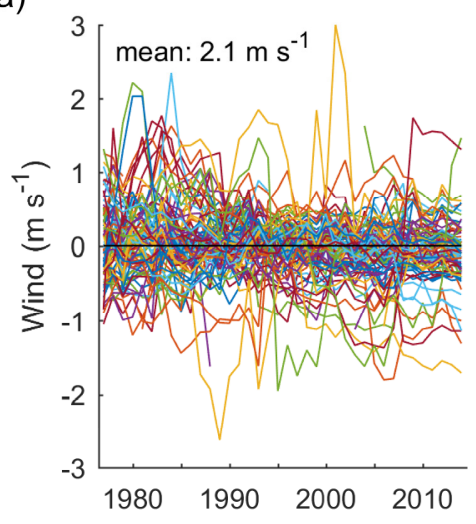

(b)

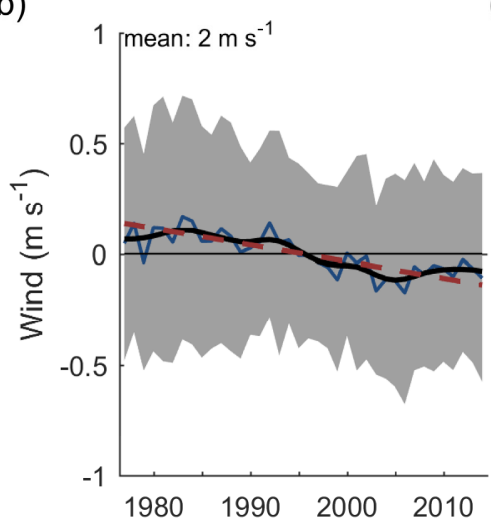

(c)

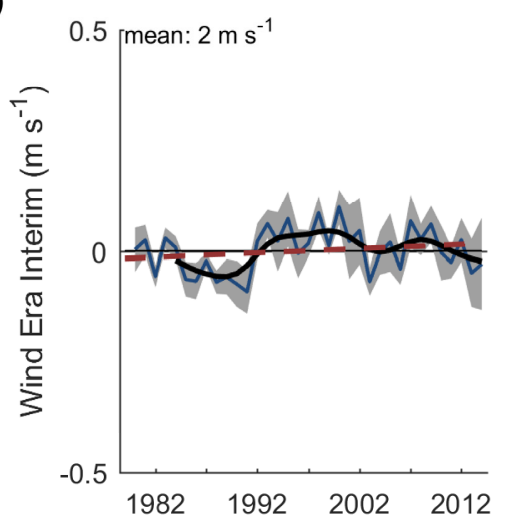

Supplementary Figure S 1 Anomalies of wind speeds (a, b) from station data (85 stations) over 1977-2014 (c) from ERA Interim data over Austria (27 grid points) over 1980-2014. (a) Each line refers to one station. (b, c) The thin blue line shows the mean over all catchments, the grey shaded area the variability between catchments $( \pm 1$ standard deviation), the bold black line the smoothed mean, and the red dashed line a trend line.

(a)

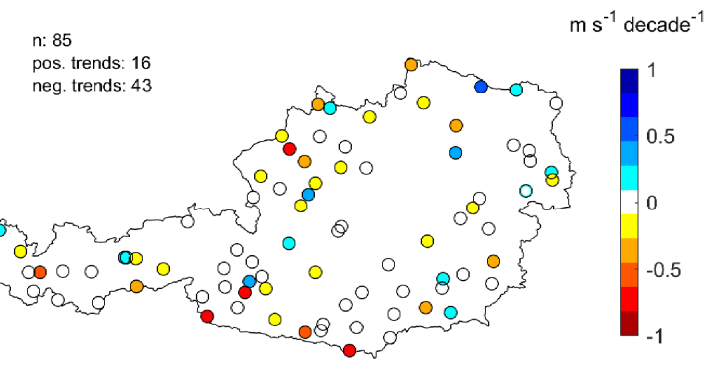

(b)

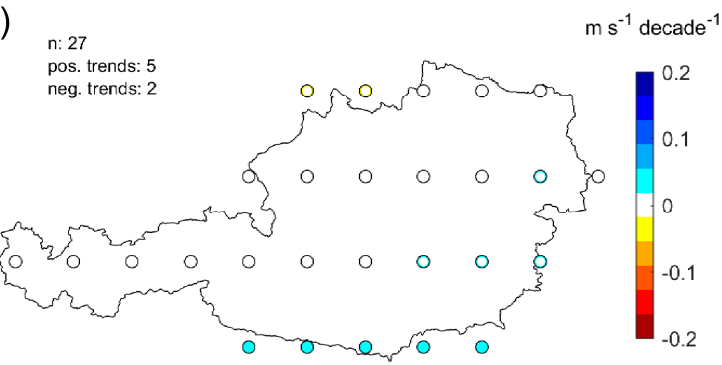

Supplementary Figure S 2: Spatial pattern of trends in wind speed (a) from 85 stations over 1977-2014 (b) from ERA Interim data over Austria over 1980-2014. Filled circles indicate significant trends at $p \leq 0.05$. 
Supplementary Table S 1 Trends in summer (May-Oct) pan evaporation of individual stations for three periods. The table includes the mean, standard deviation (Std), and coefficient of variation (CV) of summer pan evaporation over the available period.

Stars indicate trend significance: $* * * p \leq 0.01, * * p \leq 0.05, * p \leq 0.1$

\begin{tabular}{|c|c|c|c|c|c|c|c|c|c|c|c|c|c|}
\hline \multirow[b]{2}{*}{ Name } & \multirow[b]{2}{*}{ Source } & \multirow[b]{2}{*}{$\begin{array}{l}\text { Elev. } \\
\text { (m) }\end{array}$} & \multirow[b]{2}{*}{$\begin{array}{l}\text { East } \\
\left({ }^{\circ}\right)\end{array}$} & \multirow[b]{2}{*}{$\begin{array}{c}\text { North } \\
\left({ }^{\circ}\right)\end{array}$} & \multirow[b]{2}{*}{$\begin{array}{l}\text { Mean } \\
(\mathrm{mm})\end{array}$} & \multirow[b]{2}{*}{$\begin{array}{l}\text { Std } \\
(\mathrm{mm})\end{array}$} & \multirow[b]{2}{*}{$\begin{array}{l}C V \\
(-)\end{array}$} & \multirow[b]{2}{*}{$\begin{array}{l}\text { Year } \\
\text { start }\end{array}$} & \multirow[b]{2}{*}{$\begin{array}{l}\text { Year } \\
\text { end }\end{array}$} & \multirow[b]{2}{*}{ Missing data } & \multicolumn{3}{|c|}{ Trend $\left(\%\right.$ decade $\left.^{-1}\right)$} \\
\hline & & & & & & & & & & & $\begin{array}{l}1979- \\
2005\end{array}$ & $\begin{array}{l}1983- \\
2015\end{array}$ & $\begin{array}{l}1993- \\
2015\end{array}$ \\
\hline Elmen-Martinau & HZB Tirol & 954 & 10.54 & 47.36 & 348 & 43 & 0.12 & 1982 & 2014 & 1983 & -0.5 & -1 & 3.1 \\
\hline Leutasch-Kirchplatzl & HZB Tirol & 1135 & 11.14 & 47.37 & 373 & 41 & 0.11 & 1982 & 2014 & 2007 & 0.6 & $5.9^{* *}$ & $12.2^{* * *}$ \\
\hline Ladis-Neuegg & HZB Tirol & 1350 & 10.65 & 47.10 & 400 & 39 & 0.10 & 1982 & 2014 & & 5.7 & 2.6 & -4.4 \\
\hline St.Johann & HZB Tirol & 667 & 12.44 & 47.52 & 336 & 51 & 0.15 & 1982 & 2014 & & $12.0^{* * *}$ & $13.1^{* * *}$ & $13.5^{* * *}$ \\
\hline Aschau & HZB Tirol & 1005 & 12.31 & 47.38 & 290 & 34 & 0.12 & 1982 & 2014 & & 0.2 & -2.6 & $-3.1^{*}$ \\
\hline Stetten & HZB Tirol & 179 & 16.38 & 48.37 & 369 & 63 & 0.17 & 1992 & 2014 & & - & - & 9.3 \\
\hline Franzensdorf & HZB Tirol & 152 & 16.64 & 48.19 & 382 & 52 & 0.14 & 1992 & 2014 & 2010,2011 & - & - & -2.9 \\
\hline Hochberg & HZB Tirol & 1672 & 12.36 & 46.82 & 373 & 44 & 0.12 & 1983 & 2014 & 2008 & $13.8^{* * *}$ & $6.2^{* * *}$ & 3.4 \\
\hline Prägraten & HZB Tirol & 1340 & 12.38 & 47.02 & 346 & 37 & 0.11 & 1983 & 2014 & & $10.8^{* * *}$ & $4.4^{*}$ & -1.2 \\
\hline Matrei & HZB Tirol & 1040 & 12.54 & 47.00 & 317 & 48 & 0.15 & 1983 & 2013 & 2010 & - & $12.2^{* * *}$ & $10.1^{* * *}$ \\
\hline Waidring & HZB NÖ & 775 & 12.55 & 47.59 & 303 & 50 & 0.17 & 1993 & 2014 & 2011 & - & - & $14.3^{* *}$ \\
\hline Lunz & HZB NÖ & 611 & 15.07 & 47.86 & 292 & 48 & 0.17 & 1992 & 2014 & 2010,2013 & - & - & -2.1 \\
\hline Frankenfels & HZB NÖ & 468 & 15.33 & 47.98 & 293 & 61 & 0.21 & 1993 & 2014 & 2007 & - & - & $22.5^{* * *}$ \\
\hline Ottenstein & HZB NÖ & 554 & 15.34 & 48.58 & 336 & 35 & 0.10 & 1994 & 2014 & & - & - & 5.9 \\
\hline Pyhra & HZB NÖ & 298 & 15.70 & 48.15 & 404 & 44 & 0.11 & 1993 & 2014 & 2000,2001 & - & - & 4.4 \\
\hline Hollenthon & HZB NÖ & 685 & 16.26 & 47.59 & 369 & 53 & 0.14 & 1993 & 2014 & & - & - & 6.5 \\
\hline Retz & ZAMG & 242 & 15.95 & 48.77 & 424 & 63 & 0.15 & 1975 & 2005 & $1987,1995,1996,1997,1998$ & 6.9 & - & - \\
\hline Schwarzenau & ZAMG & 500 & 15.27 & 48.75 & 337 & 38 & 0.11 & 1975 & 2001 & $1985,1995,1997,1998,1999$ & - & - & - \\
\hline Hörsching & ZAMG & 298 & 14.19 & 48.24 & 442 & 60 & 0.14 & 1978 & 2005 & $1995,1998,2004$ & 5.2 & - & - \\
\hline Wien & ZAMG & 163 & 16.40 & 48.25 & 470 & 73 & 0.16 & 1979 & 2005 & $1994,1995,1997,1998$ & $12.6 * * *$ & - & - \\
\hline Innsbruck Flugh. & ZAMG & 579 & 11.36 & 47.26 & 459 & 64 & 0.14 & 1976 & 2005 & $1995,1998,2002$ & 6.4 & - & - \\
\hline Vandans & ZAMG & 670 & 9.86 & 47.09 & 317 & 47 & 0.15 & 1979 & 2005 & $1983,1995,1998,1999,2000$ & 6.8 & - & - \\
\hline Zeltweg & ZAMG & 669 & 14.78 & 47.20 & 389 & 63 & 0.16 & 1978 & 2004 & $1981,1982,1984,1995,1998$ & - & - & - \\
\hline Klagenfurt & ZAMG & 447 & 14.33 & 46.65 & 454 & 81 & 0.18 & 1976 & 2005 & $1978,1991,1995,1996,1998,2000$ & $13.8^{* *}$ & - & - \\
\hline
\end{tabular}




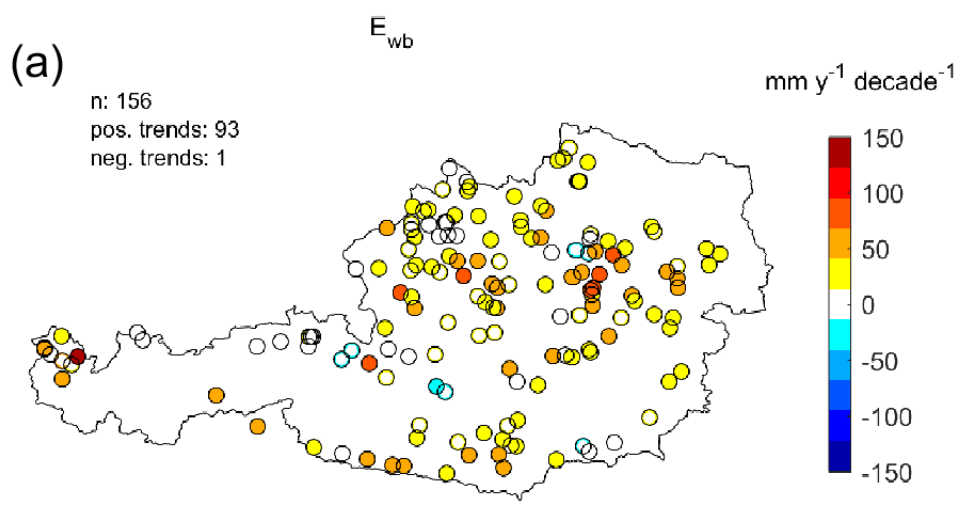

(b)

Precipitation

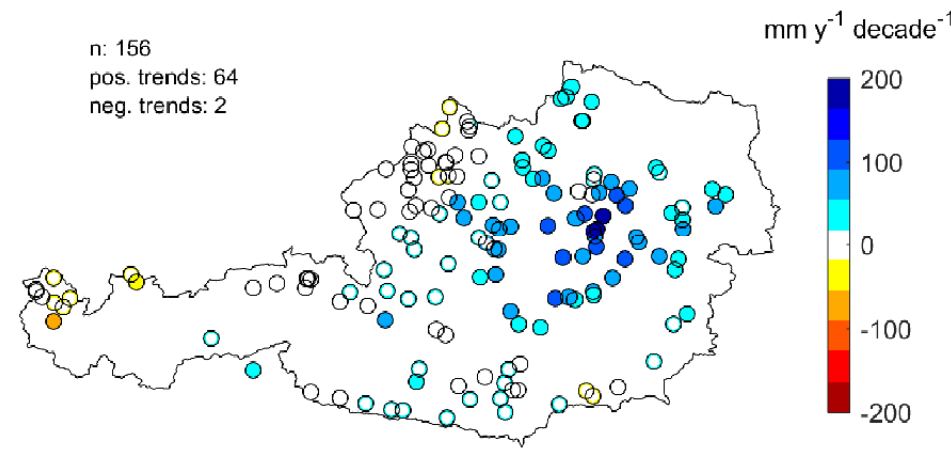

(c) Q

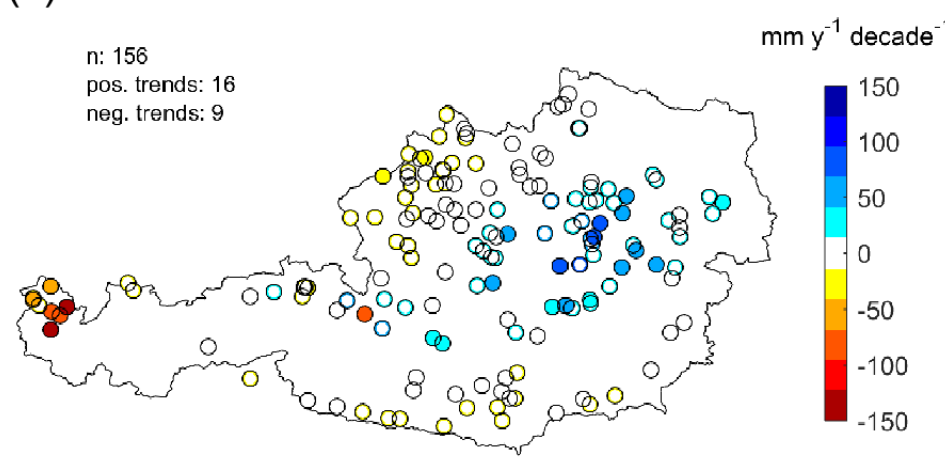

Supplementary Figure S 3 Spatial pattern of trends in (a) $E_{\mathrm{wb}}$, (b) precipitation and (c) discharge over 1977-2014. Each circle indicates the outlet of one catchment. Filled circles indicate significant trends at $p \leq 0.05$. 
(a)

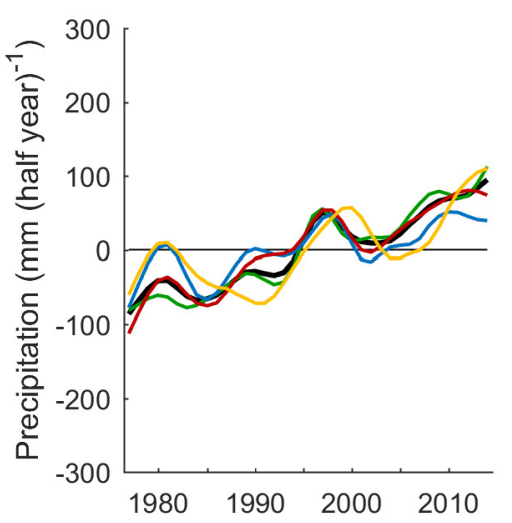

(c)
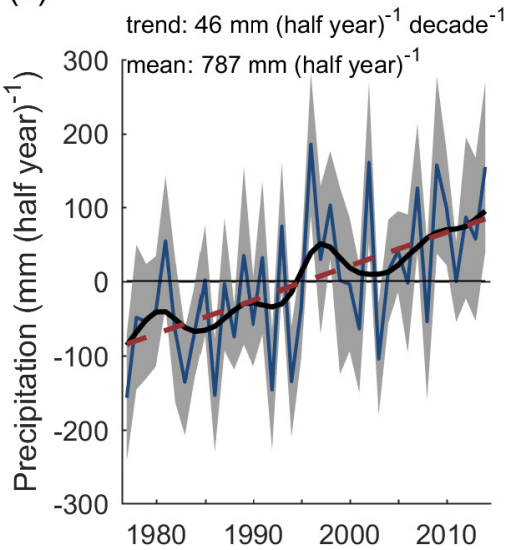

(b)

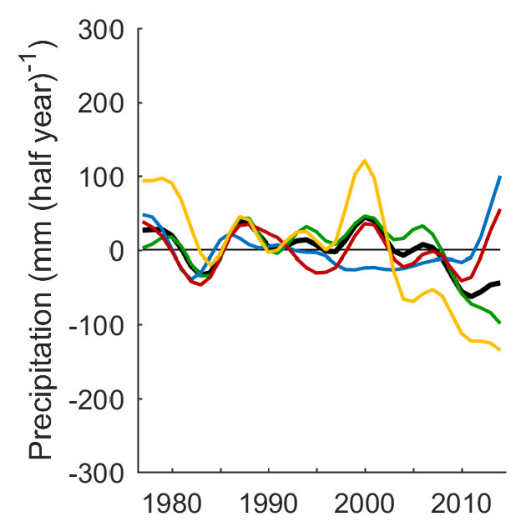

(d)

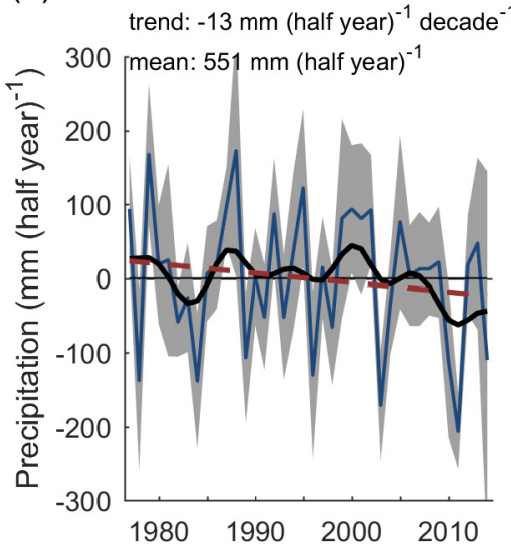

Supplementary Figure S 4 Anomalies of (a, c) summer precipitation (May-Oct), (b, d) winter precipitation (Nov-Apr) over 19772014. (a)-(b) mean anomalies by region. Data smoothed using a Gaussian filter with a standard deviation of 2 years. (c)-(d) mean anomalies over all catchments. The thin blue line shows the mean over all catchments, the grey shaded area the variability between catchments ( \pm 1 standard deviation), the bold black line the smoothed mean, and the red dashed line the trend.

(a)

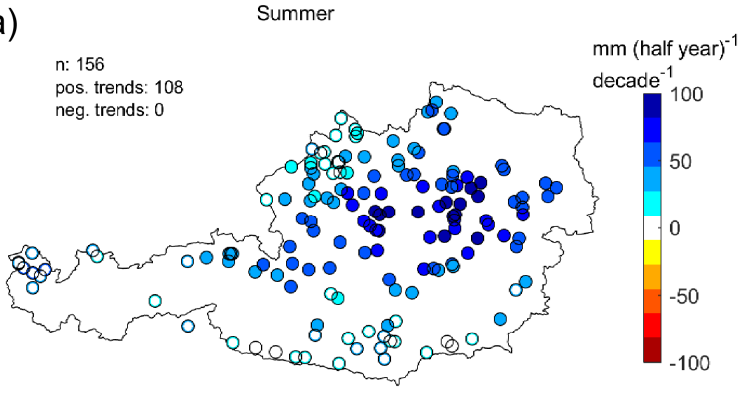

(b)

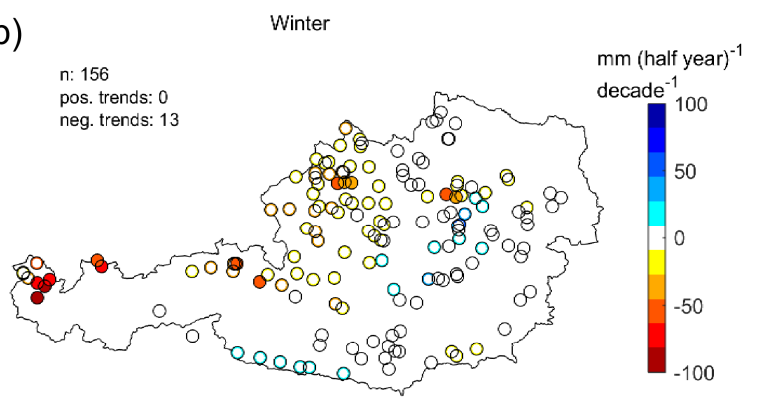

Supplementary Figure S 5 Spatial pattern of trends in (a) summer precipitation (May-Oct), and (b) winter precipitation (Nov-Apr) over 1977-2014. Each circle indicates the outlet of one catchment. Filled circles indicate significant trends at $p \leq 0.05$. 
(a)

1979-2005

n: 13

pos. trends: 5

neg. trends: 0

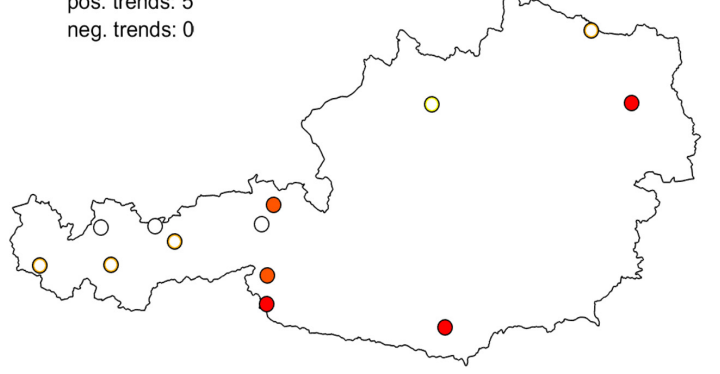

(c)

1993-2014

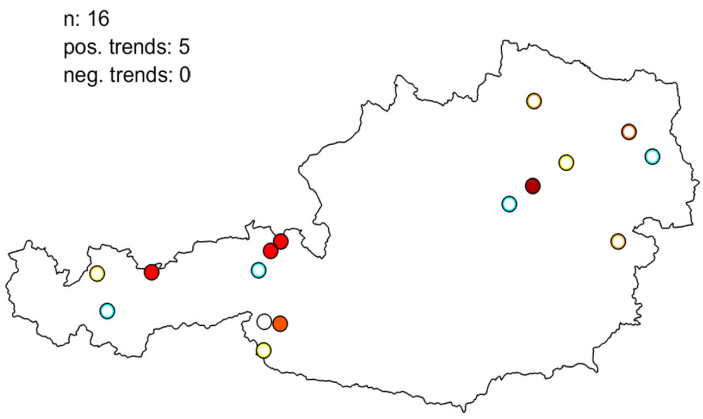

n: 16

pos. trends: 5

$0\}$ (b)

1983-2014

$$
\begin{aligned}
& \text { n: } 8 \\
& \text { pos. trends: } 4 \\
& \text { neg. trends: } 0
\end{aligned}
$$

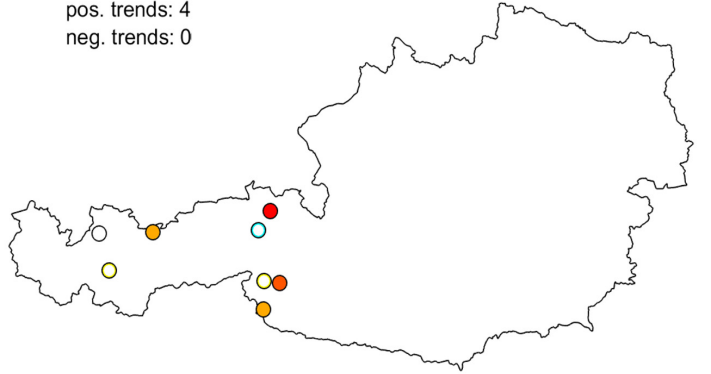

$\%$ decade $^{-1}$

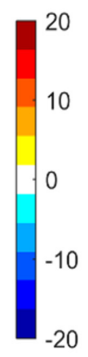

Supplementary Figure S 6 Trends in summer pan evaporation over three different periods. Filled circles indicate significant trends at $\boldsymbol{p} \leq \mathbf{0 . 0 5}$. 

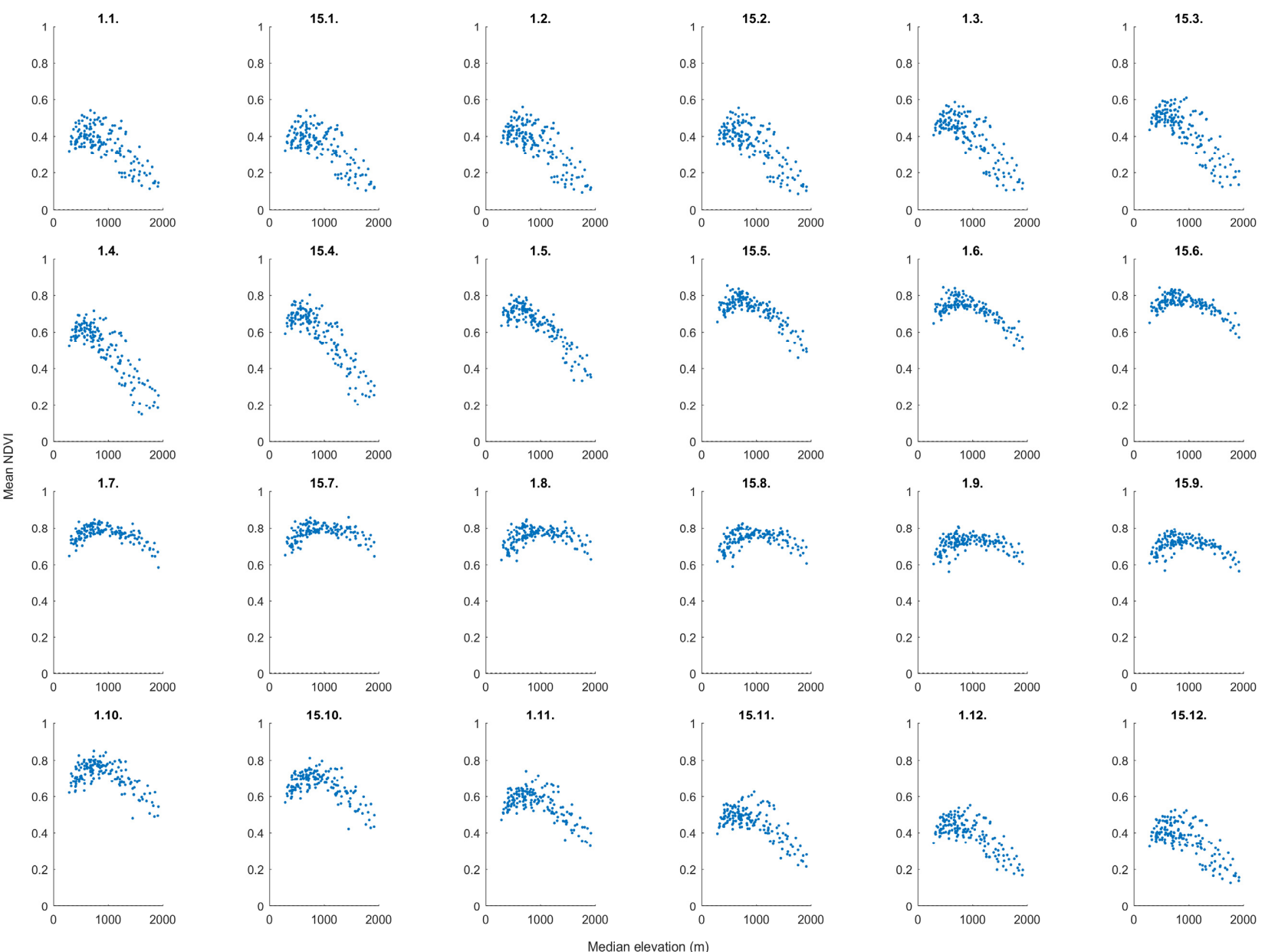

Supplementary Figure S 7 Scatterplots of catchment average NDVI ( $y$-axis) versus median catchment elevation ( $x$-axis) over the course of the year (different plots). 


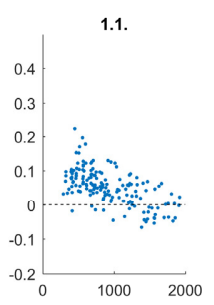

1.4 .
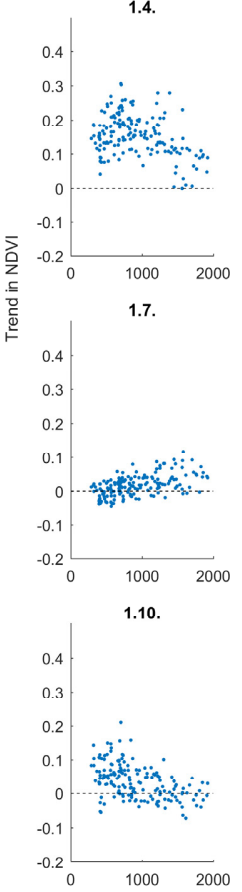

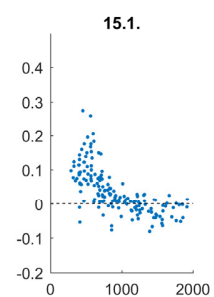

15.4.

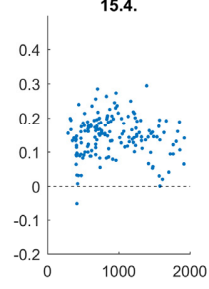

15.7

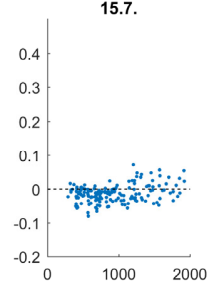

15.10.

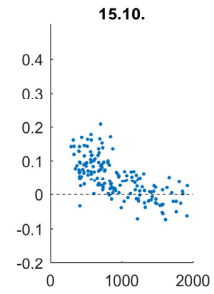

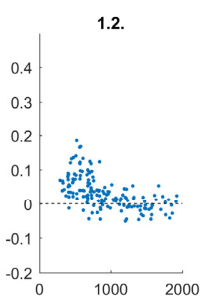

1.5.
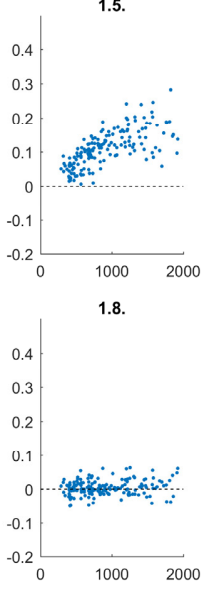

1.11

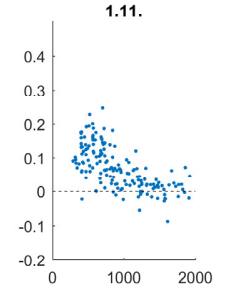

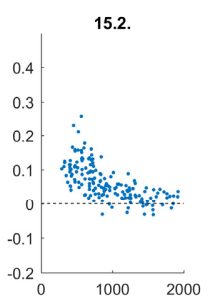

15.5.

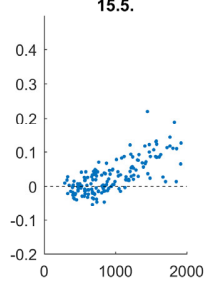

15.8.

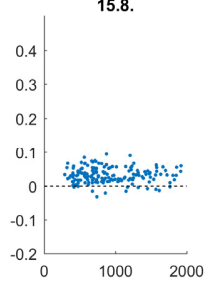

15.11 .

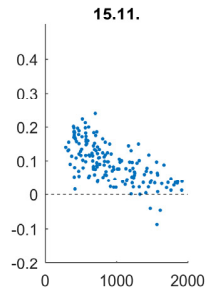

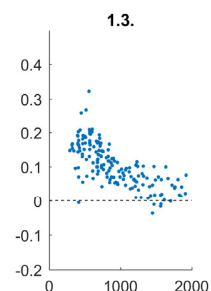

1.6 .

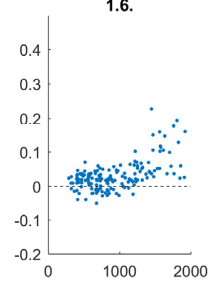

1.9.

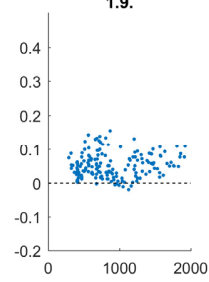

1.12.

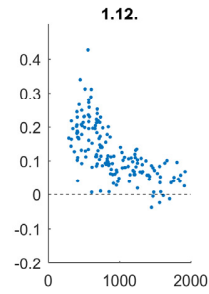

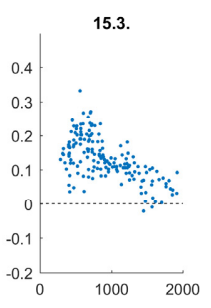

15.6 .

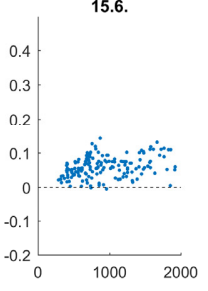

15.9

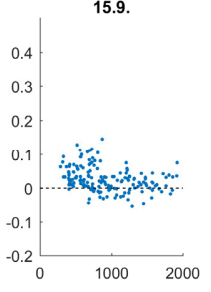

5.12.

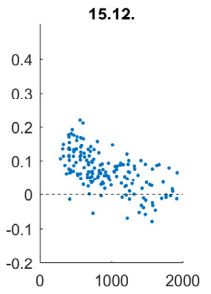

Supplementary Figure S 8 Scatterplots of catchment average NDVI trend over 1982-2014 ( $y$-axis) versus median catchment elevation ( $x$-axis) over the course of the year (different plots).

\section{References}

Böhm, R.: Heisse Luft: Reizwort Klimawandel: Fakten, Ängste, Geschäfte, Ed. Va Bene, 2008.

Dee, D. P., Uppala, S. M., Simmons, A. J., Berrisford, P., Poli, P., Kobayashi, S., Andrae, U., Balmaseda, M. A., Balsamo, G., Bauer, P., Bechtold, P., Beljaars, A. C. M., van de Berg, L., Bidlot, J., Bormann, N., Delsol, C., Dragani, R., Fuentes, M., Geer, A. J., Haimberger, L., Healy, S. B., Hersbach, H., Holm, E. V., Isaksen, L., Kallberg, P., Kohler, M., Matricardi, M., McNally, A. P., Monge-Sanz, B. M., Morcrette, J. J., Park, B. K., Peubey, C., de Rosnay, P., Tavolato, C., Thepaut, J. N., and Vitart, F.: The ERA-Interim reanalysis: configuration and performance of the data assimilation system, Q. J. Roy. Meteor. Soc., 137, 553-597, 10.1002/qj.828, 2011. 